University of Nebraska - Lincoln DigitalCommons@University of Nebraska - Lincoln

1987

\title{
Construction of Explicit and Implicit Symmetric TVD Schemes and Their Applications
}

Helen C. Yee

NASA Ames Research Center, yee@nas.nasa.gov

Follow this and additional works at: http://digitalcommons.unl.edu/nasapub

Yee, Helen C., "Construction of Explicit and Implicit Symmetric TVD Schemes and Their Applications" (1987). NASA Publications. 283.

http://digitalcommons.unl.edu/nasapub/283

This Article is brought to you for free and open access by the National Aeronautics and Space Administration at DigitalCommons@University of Nebraska - Lincoln. It has been accepted for inclusion in NASA Publications by an authorized administrator of DigitalCommons@University of Nebraska - Lincoln. 


\title{
Construction of Explicit and Implicit Symmetric TVD Schemes and Their Applications*
}

\author{
H. C. $\mathrm{YEE}^{\dagger}$ \\ NASA Ames Research Center, Moffett Field, California, 94035 \\ Received July 31, 1985; revised April 16, 1986
}

\begin{abstract}
A one-parameter family of second-order explicit and implicit total variation diminishing (TVD) schemes is reformulated so that a simplier and wider group of limiters is included. The resulting scheme can be viewed as a symmetrical algorithm with a variety of numerical dissipation terms that are designed for weak solutions of hyperbolic problems. This is a generalization of recent works of Roe and Davis to a wider class of symmetric schemes other than Lax-Wendroff. The main properties of the present class of schemes are that they can be implicit, and, when steady-state calculations are sought, the numerical solution is independent of the time step. Numerical experiments with two-dimensional unsteady and steady-state airfoil calculations show that the proposed symmetric TVD schemes are quite robust and accurate. (1) 1987 Academic Press, Inc.
\end{abstract}

\section{INTRODUCTION}

The notion of total variation diminishing (TVD) schemes was introduced Harten $[1,2]$. He derived a set of sufficient conditions which is very useful checking or constructing second-order TVD schemes. The main mechanism tha currently in use for satisfying TVD sufficient conditions involves some kind limiting procedure. There are generally two types of limiters: namely slope limi [3] and flux limiters [4-6]. For a slope limiter one imposes constraints on gradients of the dependent variables. In contrast, for a flux limiter one imposes $c$ straints on the gradients of the flux functions. For constant coefficients, the types of limiters are equivalent. The main property of a TVD scheme is that, un monotone schemes, it can be second-order accurate and is oscillation-free acs discontinuities (when applied to nonlinear scalar hyperbolic conservation laws constant coefficient hyperbolic systems). Sweby [5] and Roe [6] constructe class of limiters as a function of the gradient ratio. Most of the current limiter use are equivalent to members of this class.

* Part of the results were included in the proceedings of the IMA Workshop on "Oscillation Tht Computations, and Methods of Compensated Compactness," University of Minnesota, April 1-6, 1 and in the Proceedings of the 6th GAMM Conference on Numerical Methods in Fluid Mechanics, ' tingen, West Germany, September 25-27, 1985.

${ }^{\dagger}$ Research Scientist, Computational Fluid Dynamics Branch. 
Although TVD schemes are designed for transient applications, they also have been applied to steady-state problems [6-10]. It is well known that explicit methods are usually easier to program and often require less storage than implicit methods, but can suffer a loss of efficiency when the time step is restricted by stability rather than accuracy. It is also commonly known that it is not very useful to extend Lax-Wendroff-type schemes to implicit methods, since the resulting schemes are not suitable for steady-state calculations. This is due to the fact that the steady-state solution will depend on the time step. Roe has recently proposed a very enlightening generalized formulation of TVD Lax-Wendroff schemes [11]. Roe's result, in turn, is a generalization of Davis's work [12]. It was the investigation of these schemes which prompted the work of this paper. Their formulation has great potential for transient applications, but as it stands is not suitable for an extension to implicit methods.

The aim of this paper is to incorporate the results of Roe [11] and Davis [12] with minor modification to a one-parameter family of explicit and implicit TVD schemes $[2,8,9]$ so that a wider group of limiters can be represented in a general but rather simple form which is at the same time suitable for steady-state applications. The final scheme can be interpreted as a three-point, spatially central difference explicit or implicit scheme which has a whole variety of more rational numerical dissipation terms than the classical way of handling shock-capturing algorithms. In other words, it is a symmetric (or non-upwind) TVD scheme. It is emphasized here that the generic use of the notion upwind and symmetric TVD schemes here pertains to the schemes without the limiter present. With a limiter present, an upwind TVD scheme no longer has its traditional upwinding meaning. The same situation also applies to symmetric TVD scheme. Another way of distinguishing an upwind from a symmetric TVD scheme is that the numerical dissipation term corresponding to an upwind TVD scheme is upwind weighted [1-3, $5-10]$, as opposed to the numerical dissipation term corresponding to a symmetric TVD scheme which is centered $[11,12]$.

The proposed scheme can be used for time-accurate or steady-state calculations. Moreover, the formulations of Roe and Davis can be considered as a member of the explicit scheme. By writing the scheme in terms of numerical fluxes with two input parameters (one for the choice of the time-differencing method and one for the option of choosing the Lax-Wendroff flux), a single computer program can easily be coded to include all of the schemes under discussion. Various limiters can be considered as external functions inside the computer program. Extension of the schemes to nonlinear scalar and system of hyperbolic conservation laws is discussed in detail. Formulation and extension of the schemes in multidimensional curvilinear coordinates can be found in references $[13,14]$.

For problems containing shocks only, numerical experiments for steady-state calculations show that the implicit symmetric TVD schemes are just as accurate as an implicit upwind TVD scheme, originally developed by Harten [2] and modified by Yee [9], while requiring less computational effort. However, for problems containing both shocks and contact discontinuities, numerical experiments show that 
the explicit symmetric TVD schemes are slightly more diffusive than the explicit upwind TVD scheme especially at the contact surfaces. Numerical examples will be given in Section 5. The effort involved in modifying some existing central difference computer codes for systems of hyperbolic conservation laws is fairly simple and straightforward. The proposed algorithms should have the potential of improving the robustness and accuracy of many practical physical and engineering calculations.

\section{Preliminaries}

In this section a class of explicit and implicit TVD schemes [2] is reviewed. Harten's sufficient conditions for this class of schemes are also stated. This set of conditions is then utilized in the subsequent sections to construct and reformulate the second-order explicit and implicit TVD schemes of Harten [1,2].

Consider the scalar hyperbolic conservation law

$$
\frac{\partial u}{\partial t}+\frac{\partial f(u)}{\partial x}=0
$$

where $f$ is the flux and $a(u)=\partial f / \partial u$ is the characteristic speed. Let $u_{j}^{n}$ be the numerical solution of (2.1) at $x=j \Delta x$ and $t=n \Delta t$, with $\Delta x$ the spatial mesh size and $\Delta t$ the time step. Consider a one-parameter family of five-point difference schemes in conservation form

$$
u_{j}^{n+1}+\lambda \theta\left(h_{j+1 / 2}^{n+1}-h_{j-1 / 2}^{n+1}\right)=u_{j}^{n}-\lambda(1-\theta)\left(h_{j+1 / 2}^{n}-h_{j-1 / 2}^{n}\right),
$$

where $0 \leqslant \theta \leqslant 1, \lambda=\Delta t / \Delta x, h_{j \pm 1 / 2}^{n}=h\left(u_{j \mp 1}^{n}, u_{j}^{n}, u_{j \pm 1}^{n}, u_{j \pm 2}^{n}\right)$, and $h_{j \pm 1 / 2}^{n+1}=h\left(u_{j \mp 1}^{n+1}\right.$, $\left.u_{j}^{n+1}, u_{j \pm 1}^{n+1}, u_{j \pm 2}^{n+1}\right)$. The function $h_{j+1 / 2}$ is commonly called a numerical flux function. Let

$$
\bar{h}_{j+1 / 2}=(1-\theta) h_{j+1 / 2}^{n}+\theta h_{j+1 / 2}^{n+1}
$$

be another numerical flux function. Then (2.2) can be rewritten as

$$
u_{j}^{n+1}=u_{j}^{n}-\lambda\left(\bar{h}_{j+1 / 2}-\bar{h}_{j-1 / 2}\right) \text {. }
$$

This numerical flux is a function of eight variables, $\bar{h}_{j+1 / 2}=\bar{h}\left(u_{j-1}^{n}, u_{j}^{n}, u_{j+1}^{n}, u_{j+2}^{n}\right.$, $\left.u_{j-1}^{n+1}, u_{j}^{n+1}, u_{j+1}^{n+1}, u_{j+2}^{n+1}\right)$, and is consistent with the conservation law (2.1) in the following sense

$$
\bar{h}(u, u, u, u, u, u, u, u)=f(u) .
$$

This one-parameter family of schemes contains implicit as well as explicit schemes. When $\theta=0,(2.2)$ is an explicit method. When $\theta \neq 0,(2.2)$ is an implicit scheme. For example, if $\theta=\frac{1}{2}$, the time-differencing is the trapezoidal formula, and, 
if $\theta=1$, the time-differencing is the backward Euler method. To simplify the notation, rewrite equation $(2.2)$ as

$$
L \cdot u^{n+1}=R \cdot u^{n},
$$

where $i$ and $R$ are the following finite-difference operators:

$$
\begin{aligned}
& (L \cdot u)_{j}=u_{j}+\lambda \theta\left(h_{j+1 / 2}-h_{j-1 / 2}\right), \\
& (R: u)_{j}=u_{j}-\lambda(1-\theta)\left(h_{j+1 / 2}-h_{j-1 / 2}\right) .
\end{aligned}
$$

The total variation of a mesh function $u^{n}$ is defined to be

$$
T V\left(u^{n}\right)=\sum_{j=-\infty}^{\infty}\left|u_{j+1}^{n}-u_{j}^{n}\right|=\sum_{j=-\infty}^{\infty}\left|\Delta_{j+1 / 2} u^{n}\right|,
$$

where $\Delta_{j+1 / 2} u^{n}=u_{j+1}-u_{j}$. Here the general convention

$$
\Delta_{j+1 / 2} z=z_{j+1}-z_{j}
$$

for any mesh function $z$ is used. The numerical scheme (2.2) for an initial-value problem of $(2.1)$ is said to be TVD if

$$
T V\left(u^{n+1}\right) \leqslant T V\left(u^{n}\right) .
$$

The following sufficient conditions for (2.2) to be a TVD scheme are due to Harten [2],

$$
T V\left(R \cdot u^{n}\right) \leqslant T V\left(u^{n}\right)
$$

and

$$
T V\left(L \cdot u^{n+1}\right) \geqslant T V\left(u^{n+1}\right) .
$$

Assume the numerical flux $h$ in (2.2) is Lipschitz continuous and (2.2) can be written as

$$
\begin{aligned}
u_{j}^{n+1} & -\lambda \theta\left(\tilde{C}_{j+1 / 2}^{-} \Delta_{j+1 / 2} u-\tilde{C}_{j-1 / 2}^{+} \Delta_{j-1 / 2} u\right)^{n+1} \\
& =u_{j}^{n}+\lambda(1-\theta)\left(\tilde{C}_{j+1 / 2}^{-} \Delta_{j+1 / 2} u-\tilde{C}_{j-1 / 2}^{+} \Delta_{j-1 / 2} u\right)^{n},
\end{aligned}
$$

where $\widetilde{C}_{j \pm 1 / 2}^{\mp}=\widetilde{C}^{\mp}\left(u_{j}, u_{j \pm 1}, u_{j \pm 2}\right)$ or possibily $\widetilde{C}_{j \pm 1 / 2}^{\mp}=\widetilde{C}^{\mp}\left(u_{j \mp 1}, u_{j}, u_{j \pm 1}, u_{j \pm 2}\right)$ are some bounded functions. Then Harten further showed that sufficient conditions for (2.11) are

(a) if for all $j$

$$
\begin{aligned}
C_{j+1 / 2}^{ \pm} & =\lambda(1-\theta) \tilde{C}_{j+1 / 2}^{ \pm} \geqslant 0, \\
C_{j+1 / 2}^{+}+C_{j+1 / 2}^{-} & =\lambda(1-\theta)\left(\tilde{C}_{j+1 / 2}^{+}+\tilde{C}_{j+1 / 2}^{-}\right) \leqslant 1,
\end{aligned}
$$


and

(b) if for all $j$

$$
-\infty<C \leqslant-\lambda \theta \tilde{C}_{j+1 / 2}^{ \pm} \leqslant 0
$$

for some finite $C$. Conditions (2.13) and (2.14) are very useful in guiding the construction of second-order-accurate TVD schemes which do not exhibit the spurious oscillation associated with the more classical second-order schemes.

Harten [1,2], Yee et al. and Yee [7-10] investigated a particular form of $C^{ \pm}$. They have shown in a variety of numerical tests that the scheme is quite useful for gas-dynamic calculations. Recently, Davis [12] derived a TVD Lax-Wendroff scheme by first rewriting an upwind TVD scheme of Sweby [5] into two terms. One term consisted of the regular Lax-Wendroff method and the other a numerical dissipation term. He then simplified the method by eliminating the upwind weighting of the dissipation term. The resulting scheme is a symmetric TVD scheme which is slightly more diffusive than its upwind TVD counterpart. It is emphasized here that, in general, a second-order upwind TVD scheme can be written as a central difference scheme plus a numerical dissipation term $[2,12,15,16]$. However, in this case, the amount of numerical dissipation is a function of the sign of the characteristic speed [15]; i.e., it is upwind weighted.

More recently, Roe [11] reformulated Davis's scheme in a way which is easier to analyze and included a class of TVD schemes not observed by Davis. The scheme by Davis becomes a special case of Roe's. The main feature of Roe's current work is that he suggested a wider class of flux limiters for the Lax-Wendroff-type of TVD schemes which with a minor modification is found to have an immediate application to scheme (2.2). The details will be discussed in the next two sections.

\section{A Generalized Formulation of a Class of Symmetric Schemes}

In this section, Roe's generalized formulation of Davis's TVD Lax-Wendroff scheme is reviewed. Then, with a minor modification, his numerical flux is shown to be applicable to a larger class of symmetric schemes. Sufficient conditions for this new class of schemes to be TVD are derived for both the constant coefficient and nonlinear scalar hyperbolic equations.

\subsection{Roe's Generalized TVD Lax-Wendroff Schemes}

Roe [11] has recently developed a generalized formulation of TVD Lax-Wendroff schemes. The form of the schemes is the usual Lax-Wendroff plus a general conservative dissipation term designed in such a way that the final scheme is TVD. For $\partial f / \partial u=a=$ constant, his scheme is written as

$$
\begin{aligned}
u_{j}^{n+1}= & u_{j}^{n}-\frac{1}{2} v(1+v) \Delta_{j-1 / 2} u-\frac{1}{2} v(1-v) \Delta_{j+1 / 2} u \\
& -\frac{1}{2}|v|(1-|v|)\left(1-Q_{j-1 / 2}\right) \Delta_{j-1 / 2} u \\
& +\frac{1}{2}|v|(1-|v|)\left(1-Q_{j+1 / 2}\right) \Delta_{j+1 / 2} u .
\end{aligned}
$$


Here $v=a \lambda=a \Delta t / \Delta x$. The first two terms represent the usual Lax-Wendroff scheme, and the other two terms represent an additional conservative dissipation. The function $Q_{j+1 / 2}$ depends on three consecutive gradients $\Delta_{j-1 / 2} u, \Delta_{j+1 / 2} u$, and $\Delta_{j+3 / 2} u$ and is of the form

$$
Q_{j+1 / 2}=Q\left(r_{j+1 / 2}^{-}, r_{j+1 / 2}^{+}\right),
$$

where

$$
r_{j+1 / 2}^{-}=\frac{\Delta_{j-1 / 2} u}{\Delta_{j+1 / 2} u}, \quad r_{j+1 / 2}^{+}=\frac{\Delta_{j+3 / 2} u}{\Delta_{j+1 / 2} u} .
$$

Here $r^{ \pm}$are not defined if $\Delta_{j+1 / 2} u=0$. One way is to add a small number to both the numerator and denominator. To avoid the use of a small parameter, or the use of extra logic in computer implementation, an equivalent representation will be discussed in the appendix. If one assumes both $Q$ and $Q / r$ are always positive, then a set of sufficient conditions for (3.1) to be TVD is

$$
\begin{aligned}
Q_{j+1 / 2} & <\frac{2}{1-|v|}, \\
\left(Q_{j+1 / 2} / r_{j+1 / 2}^{-}\right) & <\frac{2}{|v|}, \\
\left(Q_{j+1 / 2} / r_{j+1 / 2}^{+}\right) & <\frac{2}{|v|} .
\end{aligned}
$$

Some examples for the function $Q$ here after called limiters (in a slightly different definition than references $[5,6])$ are

$$
\begin{aligned}
Q\left(r^{-}, r^{+}\right)= & \operatorname{minmod}\left(1, r^{-}\right)+\operatorname{minmod}\left(1, r^{+}\right)-1, \\
Q\left(r^{-}, r^{+}\right)= & \operatorname{minmod}\left(1, r^{-}, r^{+}\right) . \\
Q\left(r^{-}, r^{+}\right)= & \operatorname{minmod}\left[2,2 r^{-}, 2 r^{+}, 0.5\left(r^{-}+r^{+}\right)\right] \\
Q\left(r^{-}, r^{+}\right)= & \max \left\{0, \min \left(2 r^{-}, 1\right), \min \left(r^{-}, 2\right)\right\} \\
& +\max \left\{0, \min \left(2 r^{+}, 1\right), \min \left(r^{+}, 2\right)\right\}-1, \\
Q\left(r^{-}, r^{+}\right)= & \frac{r^{-}+\left|r^{-}\right|}{1+r^{-}}+\frac{r^{+}+\left|r^{+}\right|}{1+r^{+}}-1 .
\end{aligned}
$$

Normally the "minmod" function of two arguments is defined as

$$
\min \bmod (x, y)=\operatorname{sgn}(x) \cdot \max \{0, \min [|x|, y \cdot \operatorname{sgn}(x)]\}
$$

but within this context

$$
\operatorname{minmod}\left(1, r^{ \pm}\right)= \begin{cases}\min \left(1, r^{ \pm}\right), & r^{ \pm}>0, \\ 0, & r^{ \pm} \leqslant 0 .\end{cases}
$$


Other forms of $Q\left(r^{-}, r^{+}\right)$are discussed in Roe [6]. Limiter (3.4d) due to Roe [6], nicknamed "superbee," is the most compressive among the above four $Q$ functions. Limiter (3.4e), due to van Leer [3] is found to produce a slightly better shock resolution than $(3.4 a)[17,5,6]$. Some study on how the various limiters affect the accuracy of numerical solutions for two-dimensional fluid dynamics applications can be found in references $[17,18]$. Here the bound on $|v|$ for limiters $(3.4 \mathrm{~d}, \mathrm{e})$ are more restrictive than $(3.4 \mathrm{a}, \mathrm{b}, \mathrm{c})$.

Scheme (3.1) is a reformulation of Davis's work [12] in a way which is easier to analyze and includes a class of TVD schemes not observed by Davis. Davis only analyzed the specific scheme (3.1) with $Q\left(r^{-}, r^{+}\right)$defined in (3.4a). The numerical flux denoted by $h_{j+1 / 2}^{L W}$ for (3.1) is

$$
h_{j+1 / 2}^{L W}=\frac{1}{2}\left\{a\left(u_{j+1}+u_{j}\right)-\left[\lambda a^{2} Q_{j+1 / 2}+|a|\left(1-Q_{j+1 / 2}\right)\right] \Delta_{j+1 / 2} u\right\} .
$$

Scheme (3.1) is second-order accurate in time and space. Observe that by setting $\theta=0$ in (2.2) and by using (3.7) as the numerical flux, the resulting scheme is (3.1).

\subsection{Schemes for Linear Scalar Hyperbolic Equations}

If one is to use (3.7) as the numerical flux for (2.2) with $\theta \neq 0$, then the resulting scheme is only useful for transient calculations. For steady-state applications, either one has to restrict the time step in a manner similar to the explicit method or the steady-state solution will depend on the time step. It is emphasized here that the dependence on the time step in steady-state solutions occurs even though the value of $\Delta t$ is similar to an explicit method. In this case $\Delta t$ is most often of the same order as $\Delta x$; thus the dependence on $\Delta t$ is less severe. The term that causes this undesirable property is the one with coefficient $\lambda$ in Eq. (3.7). Therefore, besides considering the use of (3.7) as the numerical flux for (2.2) when $\theta=0$, the numerical flux (3.7) with $\lambda a^{2} Q_{j+1 / 2}=0$ and $0 \leqslant \theta \leqslant 1$ is also considered; i.e., the numerical flux is of the form

$$
h_{j+1 / 2}=\frac{1}{2}\left[a\left(u_{j+1}+u_{j}\right)-|a|\left(1-Q_{j+1 / 2}\right) \Delta_{j+1 / 2} u\right] .
$$

Now the question is, will the new numerical flux (3.8) satisfy the sufficient conditions (2.11)? The answer is yes. It turns out that some of the $Q$ functions that are suitable for the generalized TVD Lax-Wendroff scheme are also suitable for (3.8). The implication is that if one chose the proper $Q$ function, the resulting scheme (2.2) together with (3.8) can be viewed as a symmetrical algorithm with a wide variety of numerical dissipation terms that satisfy the TVD property.

Now with the choice of (3.8), the corresponding $\tilde{C}^{ \pm}$of Eq. (2.12) are

$$
\begin{array}{lll}
\tilde{C}_{j-1 / 2}^{+}=a\left[1-\frac{1}{2} Q_{j-1 / 2}+\frac{1}{2}\left(Q_{j+1 / 2} / r_{j+1 / 2}^{-}\right)\right], & & a>0, \\
\tilde{C}_{j+1 / 2}^{-}=|a|\left[1-\frac{1}{2} Q_{j+1 / 2}+\frac{1}{2}\left(Q_{j-1 / 2} / r_{j-1 / 2}^{+}\right)\right], & & a<0 .
\end{array}
$$

Therefore, sufficient conditions for this specific numerical flux function (3.8) to be TVD are 
and

$$
\begin{array}{ll}
0<\lambda(1-\theta) a\left[1-\frac{1}{2} Q_{j-1 / 2}+\frac{1}{2}\left(Q_{j+1 / 2} / r_{j+1 / 2}^{-}\right)\right]<1, & a>0, \\
0<\lambda(1-\theta)|a|\left[1-\frac{1}{2} Q_{j+1 / 2}+\frac{1}{2}\left(Q_{j-1 / 2} / r_{j-1 / 2}^{+}\right)\right]<1, & a<0,
\end{array}
$$

$$
-\infty<-\lambda \theta \widetilde{C}_{j+1 / 2}^{ \pm} \leqslant 0 .
$$

For $0 \leqslant \theta \leqslant 1$ and $v \neq 0$, condition $(3.10 \mathrm{a})$ is satisfied if

$$
\begin{aligned}
Q_{j-1 / 2}-\left(Q_{j+1 / 2} / r_{j+1 / 2}^{-}\right) & <2, \\
\left(Q_{j+1 / 2} / r_{j+1 / 2}^{-}\right)-Q_{j-1 / 2} & <\frac{2}{\lambda(1-\theta) a}-2, \\
\lambda a & <\frac{1}{1-\theta},
\end{aligned}
$$

and condition $(3.10 \mathrm{~b})$ is satisfied if

$$
\begin{aligned}
Q_{j+1 / 2}-\left(Q_{j-1 / 2} / r_{j-1 / 2}^{+}\right) & <2, \\
\left(Q_{j-1 / 2} / r_{j-1 / 2}^{+}\right)-Q_{j+1 / 2} & <\frac{2}{\lambda(1-\theta)|a|}-2, \\
\lambda|a| & <\frac{1}{1-\theta} .
\end{aligned}
$$

Since $\lambda|a| \leqslant 1 /(1-\theta)$, the term $2 /[\lambda(1-\theta)|a|]-2$ is always positive. Therefore the same assumption as Roe can be made; i.e., assume both $Q$ and $Q / r$ are always positive. Then all one has to do is devise a function $Q$ such that

$$
\begin{aligned}
Q_{j+1 / 2} & <2, \\
\left(Q_{j+1 / 2} / r_{j+1 / 2}^{-}\right) & <\frac{2}{\lambda(1-\theta)|a|}-2, \\
\left(Q_{j+1 / 2} / r_{j+1 / 2}^{+}\right) & <\frac{2}{\lambda(1-\theta)|a|}-2, \\
\lambda|a| & <\frac{1}{1-\theta} .
\end{aligned}
$$

With the above choice of $Q$, the last sufficient condition (3.11) is immediately satisfied. Some readily available $Q_{j+1 / 2}$ functions can be found in reference [11]. For instance, the examples given in equations (3.4a)-(3.4c) satisfy condition (3.13). The $Q$ functions in (3.4) all are designed such that $Q(1,1)=1$; i.e., for the scheme to be second-order accurate on a smooth region. In this case, one further restricts condition $(3.13 \mathrm{~d})$ as follows:

$$
\lambda|a|<\frac{2}{3(1-\theta)} .
$$


For $\theta=\frac{1}{2}$, scheme (2.2) together with (3.8) and (3.13) is second-order accurate in both space and time. The CFL-like restriction for (2.2) together with (3.13e) to be TVD in this case is $\frac{4}{3}$. When $\theta=1$, scheme (2.2) together with (3.8) and (3.13) is unconditionally TVD, but the resulting scheme is first-order in time and secondorder in space. When $\theta=0$, the scheme is explicit, and unlike Roe's schemes, is only first-order in time but second-order in space. As a side remark, when $\theta=0$ and $Q=1,(3.8)$ is forward Euler in time and central difference in space and hence is unconditionally unstable. However, with a proper choice of $Q \neq 1$ and $\theta=0$, the explicit scheme is TVD.

As noted before, the value $r_{j+1 / 2}^{-}\left(\right.$or $\left.r_{j+1 / 2}^{+}\right)$is not defined if $\Delta_{j-1 / 2} u$ (or $\Delta_{j+3 / 2} u$ ) is finite and $\Delta_{j+1 / 2}=0$. For computer implementation purposes, it might be more convenient to define $Q_{j+1 / 2} \Delta_{j+1 / 2} u=\hat{Q}_{j+1 / 2}$, where $\hat{Q}_{j+1 / 2}$ is a function of $\Delta_{j-1 / 2} u$, $\Delta_{j+1 / 2} u$, and $\Delta_{j+3 / 2} u$, but not a ratio of those gradients. For this formulation, see the Appendix.

\subsection{Linearized Version of the Proposed Scheme for Constant Coefficient Equations}

For $\theta \neq 0$, scheme (2.2) is implicit. Moreover, this is a genuinely nonlinear scheme in the sense that the final algorithm is nonlinear even for the constant coefficient case. The value of $u^{n+1}$ is obtained as the solution of a system of nonlinear algebraic equations. To solve this set of nonlinear equations noniteratively, a linearized version of (2.2) together with (3.8) is considered. Substituting (3.8) in (2.2), one obtains

$$
\begin{aligned}
u_{j}^{n+1} & +\frac{\lambda \theta}{2}\left[a u_{j+1}-|a|\left(1-Q_{j+1 / 2}\right) \Delta_{j+1 / 2} u\right]^{n+1} \\
& -\frac{\lambda \theta}{2}\left[a u_{j-1}-|a|\left(1-Q_{j-1 / 2}\right) \Delta_{j-1 / 2} u\right]^{n+1}=\text { r.h.s. of }(2.2),
\end{aligned}
$$

with $h_{j+1 / 2}^{n}$ defined in (3.8). Locally linearizing the coefficients of $\left(\Delta_{j \pm 1 / 2} u\right)^{n+1}$ in (3.14) by dropping the time index from $(n+1)$ to $n$, one gets

$$
\begin{gathered}
u_{j}^{n+1}+\frac{\lambda \theta}{2}\left[a u_{j+1}^{n+1}-a u_{j-1}^{n+1}-|a|\left(1-Q_{j+1 / 2}^{n}\right) \Delta_{j+1 / 2} u^{n+1}\right. \\
\left.+|a|\left(1-Q_{j-1 / 2}^{n}\right) \Delta_{j-1 / 2} u^{n+1}\right]=\text { r.h.s. of }(2.2) .
\end{gathered}
$$

Letting $d_{j}=u_{j}^{n+1}-u_{j}^{n}$ (the "delta" notation), Eq. (3.15) can be written as

$$
e_{1} d_{j-1}+e_{2} d_{j}+e_{3} d_{j+1}=-\lambda\left(h_{j+1 / 2}^{n}-h_{j-1 / 2}^{n}\right),
$$

where

$$
e_{1}=\frac{\lambda \theta}{2}\left[-a-|a|\left(1-Q_{j-1 / 2}\right)\right]^{n}
$$




$$
\begin{aligned}
& e_{2}=1+\frac{\lambda \theta}{2}\left[|a|\left(1-Q_{j-1 / 2}\right)+|a|\left(1-Q_{j+1 / 2}\right)\right]^{n} \\
& e_{3}=\frac{\lambda \theta}{2}\left[a-|a|\left(1-Q_{j+1 / 2}\right)\right]^{n} .
\end{aligned}
$$

The linearized form (3.16) is a spatially five-point scheme and yet it is a tridiagonal system of linear equations. This is because at the $(n+1)$ th time level only three points are involved; i.e., $u_{j-1}^{n+1}, u_{j}^{n+1}$, and $u_{j+1}^{n+1}$. Although the coefficients $e_{i}$ involve five points, they are at the $n$th time level.

The form of $\tilde{C}_{j \pm 1 / 2}^{ \pm}$for (3.15) is the same as (3.9) except the time index for the $Q_{j \pm 1 / 2}$ and $r_{j+1 / 2}^{ \pm}$is dropped from $(n+1)$ to $n$ for the implicit operator. One would expect that the linearized form (3.16) is still TVD. Numerical study on one and two-dimensional gas dynamics problems supported this hypothesis. It was found in reference [7] that when time-accurate TVD schemes are used as a relaxation method for steady-state calculations, the convergence rate is degraded if limiters are present on the implicit operator. For steady-state applications, one can obtain another linearized form by setting $Q_{j \pm 1 / 2}=0$ in (3.16); i.e., by redefining (3.16) by

$$
\begin{aligned}
& e_{1}=\frac{\lambda \theta}{2}(-a-|a|), \\
& e_{2}=1+\lambda \theta|a|, \\
& e_{3}=\frac{\lambda \theta}{2}(a-|a|) .
\end{aligned}
$$

Scheme (3.16a) together with (3.17) is spatially first-order accurate for the implicit operator and spatially second-order accurate for the explicit operator. Equation (3.17) is considered because no limiter is present for the implicit operator.

\subsection{Scheme for Nonlinear Scalar Hyperbolic Conservation Laws}

To extend the scheme to nonlinear scalar problems, one simply defines a local characteristic speed

$$
a_{j+1 / 2}= \begin{cases}\Delta_{j+1 / 2} f / \Delta_{j+1 / 2} u, & \Delta_{j+1 / 2} u \neq 0 \\ \left.(\partial f / \partial u)\right|_{u j}, & \Delta_{j+1 / 2} u=0\end{cases}
$$

and redefines the $r_{j+1 / 2}^{ \pm}$in $(3.2 \mathrm{~b})$ as

$$
r_{j+1 / 2}^{-}=\frac{\left|a_{j-1 / 2}\right| \Delta_{j-1 / 2} u}{\left|a_{j+1 / 2}\right| \Delta_{j+1 / 2} u}, \quad r_{j+1 / 2}^{+}=\frac{\left|a_{j+3 / 2}\right| \Delta_{j+3 / 2} u}{\left|a_{j+1 / 2}\right| \Delta_{j+1 / 2} u} .
$$

Unlike the constant coefficient case, $a_{j+1 / 2}$ and $a_{j-1 / 2}$ are not always of the same sign. After considering all the possible combinations of the signs of the $a_{j+1 / 2}$ and 
$a_{j-1 / 2}$, a set of sufficient conditions on $Q$ still can be of a form similar to (3.13) and is

$$
\begin{aligned}
Q_{j+1 / 2} & <2, \\
\left(Q_{j+1 / 2} / r_{j+1 / 2}^{-}\right) & <\frac{2}{\lambda(1-\theta)\left|a_{j-1 / 2}\right|}-2, \\
\left(Q_{j+1 / 2} / r_{j+1 / 2}^{+}\right) & <\frac{2}{\lambda(1-\theta)\left|a_{j+3 / 2}\right|}-2, \\
\lambda\left|a_{j+1 / 2}\right| & <\frac{2}{3(1-\theta)} .
\end{aligned}
$$

It is remark that the different in indices on $a$ in equations (3.20b) and (3.20c) is not important since for $\theta \neq 1$, the CFL-like restriction is limited by (3.20d). For $\theta=1$, the scheme is unconditionally stable. The numerical flux for the nonlinear case is

$$
h_{j+1 / 2}=\frac{1}{2}\left[\left(f_{j+1}+f_{j}\right)-\left|a_{j+1 / 2}\right|\left(1-Q_{j+1 / 2}\right) \Delta_{j+1 / 2} u\right] .
$$

Observe that when $a_{j+1 / 2}=0$, the scheme has zero dissipation. One way is to approximate $\left|a_{j+1 / 2}\right|$ by a Lipschitz continuous function [2]. For example, instead of using (3.21), one can use

$$
h_{j+1 / 2}=\frac{1}{2}\left[\left(f_{j+1}+f_{j}\right)-\psi\left(a_{j+1 / 2}\right)\left(1-Q_{j+1 / 2}\right) \Delta_{j+1 / 2} u\right] .
$$

Here $\psi$ is a function of $a_{j+1 / 2}$ and is of the form

$$
\psi(z)= \begin{cases}|z|, & |z| \geqslant \varepsilon \\ \left(z^{2}+\varepsilon^{2}\right) / 2 \varepsilon, & |z|<\varepsilon\end{cases}
$$

or

$$
\psi(z)= \begin{cases}|z|, & |z| \geqslant \varepsilon \\ \varepsilon, & |z|<\varepsilon,\end{cases}
$$

where $\varepsilon$ is a positive small number [9].

\subsection{Alternate Scheme for the Nonlinear Scalar Hyperbolic Problem}

A simpler way of extending the constant coefficient case to the nonlinear case is to define a local characteristic speed $a_{j+1 / 2}$ and keep the restriction on $Q$ the same as in (3.20), but use the $r_{j+1 / 2}^{ \pm}$in (3.2b) instead of (3.19). In other words, one imposes constraints on the gradients of the dependent variables instead of the flux function. The alternate form requires less computation than the previous approach. The relative advantages and disadvantages between these two forms remain to be shown. However, numerical experiments with two-dimensional Euler equations of gas dynamics [17] show that the alternate form gives a better shock resolution than the former one (3.18)-(3.22). 
As a side remark, a case of Harten's second-order explicit TVD scheme is contained in the class of limiters of Sweby [5] and Roe [6] and is equivalent to a case of Roe's second-order scheme of reference [6]. See Sweby's original manuscript [19] instead of the published version [5] for details. The numerical experiments of Yee et al. and Yee [7-9] with Harten's type of second-order TVD scheme indicate that the alternate form is favored over the approach (3.18)-(3.22). This indication is further endorsed by Davis's numerical experiments [12] with similar examples.

\subsection{Linearized Version of the Proposed Implicit Scheme for Nonlinear Equations}

For the nonlinear case, the situation is slightly more complicated since the characteristic speed $\partial f / \partial u$ is no longer a constant. Substituting (3.22) in (2.2), one obtains

$$
\begin{aligned}
u_{j}^{n+1} & +\frac{\lambda \theta}{2}\left[f_{j+1}-\psi\left(a_{j+1 / 2}\right)\left(1-Q_{j+1 / 2}\right) \Delta_{j+1 / 2} u\right]^{n+1} \\
& -\frac{\lambda \theta}{2}\left[f_{j-1}-\psi\left(a_{j-1 / 2}\right)\left(1-Q_{j-1 / 2}\right) \Delta_{j-1 / 2} u\right]^{n+1}=\text { r.h.s. of }(2.2) .
\end{aligned}
$$

Unlike the constant coefficient case, one also has to linearize $f_{j \pm 1}^{n+1}, \psi\left(a_{j \pm 1 / 2}^{n+1}\right)$, and $Q_{j \pm 1 / 2}^{n+1}$. Following the same procedure as in [9], two linearized versions of (3.25) are considered.

Linearized Nonconservative Implicit Form. Adding and substracting $f_{j}^{n+1}$ on the left-hand side of (3.25) and using the relation (3.18), one can rewrite (3.25) as

$$
\begin{aligned}
u_{j}^{n+1} & +\frac{\lambda \theta}{2}\left[a_{j+1 / 2}^{n+1}-\psi\left(a_{j+1 / 2}^{n+1}\right)\left(1-Q_{j+1 / 2}^{n+1}\right)\right] \Delta_{j+1 / 2} u^{n+1} \\
& -\frac{\lambda \theta}{2}\left[-a_{j-1 / 2}^{n+1}-\psi\left(a_{j-1 / 2}^{n+1}\right)\left(1-Q_{j-1 / 2}^{n+1}\right)\right] \Delta_{j-1 / 2} u^{n+1}=\text { r.h.s. of }(2.2) .
\end{aligned}
$$

By dropping the time index of the coefficients of $\Delta_{j \pm 1 / 2} u^{n+1}$ from $(n+1)$ to $n(3: 26)$ becomes

$$
\bar{e}_{1} d_{j-1}+\bar{e}_{2} d_{j}+\bar{e}_{3} d_{j+1}=-\lambda\left(h_{j+1 / 2}^{n}-h_{j-1 / 2}^{n}\right)
$$

where

$$
\begin{aligned}
& \bar{e}_{1}=\lambda \theta B^{-} \\
& \bar{e}_{2}=1-\lambda \theta\left(B^{-}+B^{+}\right), \\
& \bar{e}_{3}=\lambda \theta B^{+},
\end{aligned}
$$

and

$$
B^{ \pm}=\frac{1}{2}\left[ \pm a_{j \pm 1 / 2}-\psi\left(a_{j \pm 1 / 2}\right)\left(1-Q_{j \pm 1 / 2}\right)\right]^{n}
$$


Again Eq. (3.27) is a five-point scheme, and yet the coefficient matrix associated with the $d_{j}$ 's is tridiagonal. With this linearization, the method is no longer conservative. Therefore (3.27) is only applicable for steady-state calculations. Again, a spatially first-order-accurate implicit operator similar to (3.17) can be obtained for (3.27) by setting $B^{ \pm}=\frac{1}{2}\left[ \pm a_{j \pm 1 / 2}-\psi\left(a_{j \pm 1 / 2}\right)\right]^{n}$. Since the limiter does not appear on the left-hand side, improvement in efficiency over (3.17) might be possible $[7,9]$. This reduced form is especially useful for multidimensional, nonlinear, hyperbolic conservation laws.

Linearized Conservative Implicit Form. One can obtain a linearized conservative implicit form by using a local Taylor expansion about $u^{n}$ and expressing $f^{n+1}-f^{n}$ in the following form

$$
f_{j}^{n+1}-f_{j}^{n}=a_{j}^{n}\left(u_{j}^{n+1}-u_{j}^{n}\right)+O\left(\Delta t^{2}\right),
$$

where $a_{j}^{n}=(\partial f / \partial u)_{j}^{n}$. Applying the first-order approximation of (3.28) and locally linearizing the coefficients of $\left(\Delta_{j \pm 1 / 2} u\right)^{n+1}$ in (3.25) by dropping the time index from $(n+1)$ to $n$, one gets

$$
\begin{aligned}
u_{j}^{n+1} & +\frac{\lambda \theta}{2}\left[a_{j+1}^{n} u_{j+1}^{n+1}-a_{j-1}^{n} u_{j-1}^{n+1}-\psi\left(a_{j+1 / 2}^{n}\right)\left(1-Q_{j+1 / 2}^{n}\right) \Delta_{j+1 / 2} u^{n+1}\right. \\
& \left.+\psi\left(a_{j-1 / 2}^{n}\right)\left(1-Q_{j-1 / 2}^{n}\right) \Delta_{j-1 / 2} u^{n+1}\right]=\text { r.h.s. of }(2.2) .
\end{aligned}
$$

Letting $d_{j}=u_{j}^{n+1}-u_{j}^{n}$, Eq. (3.29) can be written as

$$
e_{1} d_{j-1}+e_{2} d_{j}+e_{3} d_{j+1}=-\lambda\left(h_{j+1 / 2}^{n}-h_{j-1 / 2}^{n}\right),
$$

where

$$
\begin{aligned}
& e_{1}=\frac{\lambda \theta}{2}\left[-a_{j-1}-\psi\left(a_{j-1 / 2}\right)\left(1-Q_{j-1 / 2}\right)\right]^{n}, \\
& e_{2}=1+\frac{\lambda \theta}{2}\left[\psi\left(a_{j-1 / 2}\right)\left(1-Q_{j-1 / 2}\right)+\psi\left(a_{j+1 / 2}\right)\left(1-Q_{j+1 / 2}\right)\right]^{n}, \\
& e_{3}=\frac{\lambda \theta}{2}\left[a_{j+1}-\psi\left(a_{j+1 / 2}\right)\left(1-Q_{j+1 / 2}\right)\right]^{n} .
\end{aligned}
$$

The linearized form (3.30) is conservative and is a spatially five-point scheme with a tridiagonal system of linear equations. Scheme (3.30) is applicable to transient as well as steady-state calculations. But the form of $\widetilde{C}_{j \pm 1 / 2}^{ \pm}$for (3.30) is no longer the same as its nonlinear counterpart. As of this writing, the conservative linearlized form (3.30) has not been proven to be TVD. Yet numerical study shows that for moderate CFL numbers, (3.30) produces high-resolution shocks and nonoscillatory solutions. 
For steady-state application, one can use a spatially first-order implicit operator for (3.30) by simply setting all the $Q_{j \pm 1 / 2}=0$; i.e., redefine $(3.30 \mathrm{~b})-(3.30 \mathrm{~d})$ as

$$
\begin{aligned}
& e_{1}=\frac{\lambda \theta}{2}\left[-a_{j-1}-\psi\left(a_{j-1 / 2}\right)\right]^{n}, \\
& e_{2}=1+\frac{\lambda \theta}{2}\left[\psi\left(a_{j-1 / 2}\right)+\psi\left(a_{j+1 / 2}\right)\right]^{n}, \\
& e_{3}=\frac{\lambda \theta}{2}\left[a_{j+1}-\psi\left(a_{j+1 / 2}\right)\right]^{n} .
\end{aligned}
$$

For an upwind TVD scheme, the analogue of the first-order linearized conservative (3.31) alternating direction implicit (ADI) form [9], numerical experiments with two-dimensional steady-state airfoil calculations show that this form is the most efficient (in terms of CPU time) among the various proposed linearized methods for the case of $\theta=1$. No comparison has been made for time-accurate calculations or for any other values of $\theta$.

\section{Extension to Hyperbolic System of Conservation Laws}

Extension of the scalar scheme (3.14), (3.27), or (3.30) to systems of conservation laws can be accomplished by defining at each point a "local" system of characteristic fields, and then applying the scheme to each of the $m$ scalar characteristic equations. Here $m$ is the dimension of the hyperbolic system. Extension of the scalar implicit scheme to higher than one-dimensional systems of conservation laws (for practical calculations) can be accomplished by an alternating direction implicit (ADI) method similar to the one described in Yee et al. and Yee [7, 9]. Only the one-dimensional case will be described here.

Formal Extension. Consider a system of hyperbolic conservation laws

$$
\frac{\partial U}{\partial t}+\frac{\partial F(U)}{\partial x}=0
$$

Here $U$ and $F(U)$ are column vectors of $m$ components. Let $A=\partial F / \partial U$ and the eigenvalues of $A$ be $\left(a^{1}, a^{2}, \ldots, a^{m}\right)$. Denote $R\left(R^{-1}\right)$ as the matrices whose columns are right (left) eigenvectors of $A\left(A^{-1}\right)$. Let $U_{j+1 / 2}$ denote some symmetric average of $U_{j}$ and $U_{j+1}$ (see $[1,7,9]$ for a formula). Let $a_{j+1 / 2}^{l}, R_{j+1 / 2}, R_{j+1 / 2}^{-1}$ denote the quantities $a^{l}, R, R^{-1}$ evaluated at $U_{j+1 / 2}$. Define

$$
\alpha_{j+1 / 2}=R_{j+1 / 2}^{-1} \Delta_{j+1 / 2} U
$$

as the forward difference (or the jump) of the local characteristic variables. With the above notation, a one-parameter family of TVD schemes (2.2) in the system case can be written as

$$
U_{j}^{n+1}+\lambda \theta\left(H_{j+1 / 2}^{n+1}-H_{j-1 / 2}^{n+1}\right)=U_{j}^{n}-\lambda(1-\theta)\left(H_{j+1 / 2}^{n}-H_{j-1 / 2}^{n}\right) .
$$


The numerical flux function $H_{j+1 / 2}$ is expressed as

$$
H_{j+1 / 2}=\frac{1}{2}\left(F_{j}+F_{j+1}-R_{j+1 / 2} \Phi_{j+1 / 2}\right),
$$

where the elements of the $\Phi_{j+1 / 2}$ denoted by $\phi_{j+1 / 2}^{l}, l=1, \ldots, m$ are

$$
\phi_{j+1 / 2}^{l}=\psi\left(a_{j+1 / 2}^{l}\right)\left(1-Q_{j+1 / 2}^{l}\right) \alpha_{j+1 / 2}^{l},
$$

with $\psi(z)$ defined in (3.23), and

$$
\begin{aligned}
Q_{j+1 / 2}^{l} & =Q\left[\left(r_{j+1 / 2}^{-}\right)^{l},\left(r_{j+1 / 2}^{+}\right)^{l}\right], \\
\left(r_{j+1 / 2}^{-}\right)^{l} & =\frac{\left|a_{j-1 / 2}^{l}\right| \alpha_{j-1 / 2}^{l}}{\left|a_{j+1 / 2}^{l}\right| \alpha_{j+1 / 2}^{l}}, \\
\left(r_{j+1 / 2}^{+}\right)^{l} & =\frac{\left|a_{j+3 / 2}^{l}\right| \alpha_{j+3 / 2}^{l}}{\left|a_{j+1 / 2}^{l}\right| \alpha_{j+1 / 2}^{l}},
\end{aligned}
$$

or

$$
\begin{aligned}
& \left(r_{j+1 / 2}^{-}\right)^{l}=\frac{\alpha_{j-1 / 2}^{l}}{\alpha_{j+1 / 2}^{l},} \\
& \left(r_{j+1 / 2}^{+}\right)^{l}=\frac{\alpha_{j+3 / 2}^{l}}{\alpha_{j+1 / 2}^{l}} .
\end{aligned}
$$

Here $\alpha_{j+1 / 2}^{l}$ are the elements of (4.2). The corresponding conservative linearized form (3.30) for the system case can be expressed as

$$
E_{1} D_{j-1}+E_{2} D_{j}+E_{3} D_{j+1}=-\lambda\left(H_{j+1 / 2}^{n}-H_{j-1 / 2}^{n}\right),
$$

where

$$
\begin{aligned}
& E_{1}=\frac{\lambda \theta}{2}\left(-A_{j-1}-K_{j-1 / 2}\right)^{n} \\
& E_{2}=I+\frac{\lambda \theta}{2}\left(K_{j-1 / 2}+K_{j+1 / 2}\right)^{n}, \\
& E_{3}=\frac{\lambda \theta}{2}\left(A_{j+1}-K_{j+1 / 2}\right)^{n},
\end{aligned}
$$

with

$$
K_{j \pm 1 / 2}=\left(R \Omega R^{-1}\right)_{j \pm 1 / 2}^{n}
$$

and

$$
\Omega_{j \pm 1 / 2}=\operatorname{diag}\left[\psi\left(a_{j \pm 1 / 2}^{l}\right)\left(1-Q_{j \pm 1 / 2}^{l}\right)\right],
$$

or for the first-order left-hand side

$$
\Omega_{j \pm 1 / 2}=\operatorname{diag}\left[\psi\left(a_{j \pm 1 / 2}^{l}\right)\right] .
$$


Here $\operatorname{diag}\left(z^{l}\right)$ denotes a diagonal matrix with diagonal elements $z^{l}$. Aside from computing the right-hand side, the rest of the arithmetic involved for Eq. (4.5) is two matrix multiplications and a block tridiagonal inversion. The value of $\Omega_{j+1 / 2}$ in (4.5f) or $(4.5 \mathrm{~g})$ can be saved while calculating the right-hand side. Similarly, one can express the nonconservative linearized form (3.27) for the system case.

As a side remark, with the same procedure Roe's numerical flux in the system case can be written as

$$
H_{j+1 / 2}^{L W}=\frac{1}{2}\left(F_{j}+F_{j+1}-R_{j+1 / 2} \Phi_{j+1 / 2}^{L W}\right),
$$

where the elements of the $\Phi_{j+1 / 2}^{L W}$ denoted by $\left(\phi_{j+1 / 2}^{l}\right)^{L W}, l=1, \ldots, m$, are

$$
\left(\phi_{j+1 / 2}^{l}\right)^{L W}=\left[\lambda\left(a_{j+1 / 2}^{l}\right)^{2} Q_{j+1 / 2}^{l}+\left|a_{j+1 / 2}^{l}\right|\left(1-Q_{j+1 / 2}^{l}\right)\right] \alpha_{j+1 / 2}^{l} .
$$

Simplified Version. As one can see, the main work for scheme (4.3a) with numerical flux function (4.3b) or (4.6) is the term $R_{j+1 / 2} \Phi_{j+1 / 2}$. A similar situation is also true for the corresponding conservative or nonconservative linearized form. Since

$$
\begin{aligned}
R_{j+1 / 2} \Phi_{j+1 / 2} & =R_{j+1 / 2} \Omega_{j+1 / 2} \alpha_{j+1 / 2}=R_{j+1 / 2} \Omega_{j+1 / 2} R_{j+1 / 2}^{-1} \Delta_{j+1 / 2} U \\
& =R_{j+1 / 2} \operatorname{diag}\left[\psi\left(a_{j+1 / 2}^{l}\right)\left(1-Q_{j+1 / 2}^{l}\right)\right] R_{j+1 / 2}^{-1} \Delta_{j+1 / 2} U,
\end{aligned}
$$

if somehow one can simplify $R \Omega R^{-1}$ to be a diagonal matrix, then the current implicit scheme should be competitive in terms of operation count with the widely distributed codes such as ARC2D (version 150) of Pulliam and Steger [20] and FLO52R of Jameson et al. [21]. Both ARC2D and FLO52R use a spatially threepoint central differencing scheme with identical numerical dissipation terms, but they use different time-stepping methods for steady-state calculations.

Two possible ways of simplifying (4.7) are one suggested by Davis [12], and one suggested by Roe [11]. Davis suggested approximating (4.7) with

$$
R_{j+1 / 2} \Omega_{j+1 / 2} R_{j+1 / 2}^{-1} \approx \bar{\omega}\left(r_{j+1 / 2}^{-}, r_{j+1 / 2}^{+}\right) I,
$$

where $\bar{\omega}_{j+1 / 2}=\bar{\omega}\left(r_{j+1 / 2}^{-}, r_{j+1 / 2}^{+}\right)$is a scalar function of $r_{j+1 / 2}^{-}, r_{j+1 / 2}^{+}$and $a_{j+1 / 2}^{l}$. The symbol $I$ is a $m \times m$ identity matrix. Adapted to the current scheme, $\bar{\omega}_{j+1 / 2}$ can be expressed as

$$
\bar{\omega}_{j+1 / 2}=\psi\left(\bar{a}_{j+1 / 2}\right)\left[1-Q\left(r_{j+1 / 2}^{-}, r_{j+1 / 2}^{+}\right)\right],
$$

where

$$
\bar{a}_{j+1 / 2}=\max _{l}\left|a_{j+1 / 2}^{l}\right| \text {. }
$$

In order to not have to compute $R$ and $R^{-1}, r^{ \pm}$have to be redefined such that they are functions of gradients of the original variables $U$ instead of $\alpha_{j+1 / 2}$. Davis suggested using 


$$
\begin{aligned}
& r_{j+1 / 2}^{-}=\frac{\left(\Delta_{j-1 / 2} U, \Delta_{j+1 / 2} U\right)}{\left(\Delta_{j+1 / 2} U, \Delta_{j+1 / 2} U\right)}=\frac{\sum_{l}\left(\Delta_{j-1 / 2} u^{l}\right)\left(\Delta_{j+1 / 2} u^{l}\right)}{\sum_{l}\left(\Delta_{j+1 / 2} u^{l}\right)^{2}}, \\
& r_{j+1 / 2}^{+}=\frac{\left(\Delta_{j+3 / 2} U, \Delta_{j+1 / 2} U\right)}{\left(\Delta_{j+1 / 2} U, \Delta_{j+1 / 2} U\right)}=\frac{\sum_{l}\left(\Delta_{j+3 / 2} u^{l}\right)\left(\Delta_{j+1 / 2} u^{l}\right)}{\sum_{l}\left(\Delta_{j+1 / 2} u^{l}\right)^{2}},
\end{aligned}
$$

where $(\cdot, \cdot)$ denotes the usual inner product on the real $m$-dimensional vector space $R^{m}$.

Roe suggested that instead of using the fastest wave (4.10), one might consider the use of the strongest wave in the following sense

$$
\begin{aligned}
\bar{a}_{j+1 / 2} & =\left.\frac{\sum_{l=1}^{m} a^{l}\left(\alpha^{l}\right)^{2}}{\sum_{l=1}^{m}\left(\alpha^{l}\right)^{2}}\right|_{j+1 / 2} \\
\left(\bar{a}_{j+1 / 2}\right)^{2} & =\left.\frac{\sum_{l=1}^{m}\left(a^{l} \alpha^{\prime}\right)^{2}}{\sum_{l=1}^{m}\left(\alpha^{\prime}\right)^{2}}\right|_{j+1 / 2}
\end{aligned}
$$

For the one-dimensional Euler equation of gas dynamics (perfect gas), the $a^{l}$ are simply

$$
a^{1}=u-c, \quad a^{2}=u, \quad a^{3}=u+c,
$$

and formulae for the $\alpha^{l}$ are

$$
\begin{aligned}
& \alpha_{j+1 / 2}^{1}=\frac{1}{2 c_{j+1 / 2}^{2}}\left(\Delta_{j+1 / 2} p-\rho_{j+1 / 2} c_{j+1 / 2} \Delta_{j+1 / 2} u\right), \\
& \alpha_{j+1 / 2}^{2}=\frac{1}{c_{j+1 / 2}^{2}}\left(c_{j+1 / 2}^{2} \Delta_{j+1 / 2} \rho-\Delta_{j+1 / 2} p\right), \\
& \alpha_{j+1 / 2}^{3}=\frac{1}{2 c_{j+1 / 2}^{2}}\left(\Delta_{j+1 / 2} p+\rho_{j+1 / 2} c_{j+1 / 2} \Delta_{j+1 / 2} u\right) .
\end{aligned}
$$

Here $u$ is the velocity, $c$ is the sound speed, $p$ is the pressure, and $\rho$ is the density.

As for the $r^{ \pm}$, Roe suggested the use of $\alpha_{j \pm 1 / 2}$ instead of $\Delta_{j \pm 1 / 2} U$ in (4.11). In this case, the operation count between the local characteristic variable approach (4.5) and Roe's suggestion might be very competitive, since in Roe's suggestion one has to compute all the $\alpha_{j+1 / 2}^{l}$ anyway. Therefore, the main difference is between computing $R_{j+1 / 2} \Omega_{j+1 / 2}=R_{j+1 / 2} \operatorname{diag}\left[\psi\left(a_{j \pm 1 / 2}^{l}\right)\left(1-Q_{j \pm 1 / 2}^{l}\right)\right]$ together with (4.4), or computing equations (4.8)-(4.9) and Eq. (4.12a) or (4.12b) together with

$$
\begin{aligned}
& r_{j+1 / 2}^{-}=\frac{\left(\alpha_{j-1 / 2}, \alpha_{j+1 / 2}\right)}{\left(\alpha_{j+1 / 2}, \alpha_{j+1 / 2}\right)}=\frac{\sum_{l} \alpha_{j-1 / 2}^{l} \alpha_{j+1 / 2}^{l}}{\sum_{l}\left(\alpha_{j+1 / 2}^{l}\right)^{2}}, \\
& r_{j+1 / 2}^{+}=\frac{\left(\alpha_{j+3 / 2}, \alpha_{j+1 / 2}\right)}{\left(\alpha_{j+1 / 2}, \alpha_{j+1 / 2}\right)}=\frac{\sum_{l} \alpha_{j+3 / 2}^{l} \alpha_{j+1 / 2}^{l}}{\sum_{l}\left(\alpha_{j+1 / 2}^{l}\right)^{2}} .
\end{aligned}
$$


In summary, three approaches are suggested for the system case: (a) the more systematic approach (4.5) (from here on referred to as the local characteristic approach), (b) Davis's approach, and (c) Roe's approach. Davis's suggestion is by far the simpliest to implement and requires the least operation count. In numerical experiments with a two-dimensional shock reflection problem and a circular arc airfoil problem, Davis's approach showed good potential. Roe's suggestion, in the author's opinion, is a compromise between the local characteristic approach and Davis's approach, but requires an operation count similar to the local characteristic approach. An advantage of the current approach over the other two approaches is that (4.5) collapses into the exact scalar scheme for a constant coefficient system. The implication is that if one locally freezes the coefficients in (4.5), then the resulting constant coefficient system is TVD and convergent subject to the CFL restriction of $2 /[3(1-\theta)]$. The proof of this statement is readily available in reference [2]. The total variation definition for a vector grid function for the system case is

$$
T V(U)=\sum_{j} \sum_{l=1}^{m}\left|\alpha_{j+1 / 2}^{l}\right|
$$

In other words, the local characteristic approach (4.5) in effect uses scalar schemes on each characteristic field. For the one-dimensional Euler equation of gas dynamics, the characteristic fields consists of two nonlinear fields $u \pm c$ and a linear field $u$. The limiter used need not be the same for each field. One can even use different schemes for different fields. This leads to the following suggestion for problems containing contact discontinuities as well as shocks (e.g., complex Mach reflections problems in two dimensions):

For nonlinear fields, use a less compressive limiter such as (3.4a), (3.4b), (3.4c). For the linear fields, use a more compressive limiter such as (3.4d) to capture contact discontinuities.

Numerical experiments on two-dimensional blast wave calculations $[17,18]$ support the above statement. The result in [17] also suggests that one should not use (3.4d) for all characteristic fields. The result also shows that (3.4d) can enhance the resolution of contact surfaces but not shocks. In certain cases, (3.4d) is too compressive and might produce unphysical solutions.

\section{Numerical ResUlts}

The numerical experiments were mainly performed on the NACA0012 and NACA0018 airfoils using the local characteristic approach and $r^{ \pm}$as defined in $(4.4 c, d)$. Implementation of the present implicit symmetric TVD scheme for twodimensional steady-state calculations is described in reference [13]. Extension of the second-order explicit symmetric TVD schemes (4.6) is by the local characteristic approach and by locally one-dimensional time splitting $[14,17]$ to preserve the second-order time accuracy. 
Steady-State Calculations. Generally, for inviscid steady-state calculations, upwind TVD schemes produce sharper shocks than symmetric TVD schemes [12]. For the current implicit symmetric TVD scheme with limiter (3.4a) or (3.4b), this seems to be not the case. The symmetric method appeared to produce almost identical results as an upwind TVD scheme which was originally developed by Harten [2] and modified by Yee [9]. Here the corresponding $\phi_{j+1 / 2}^{l}$ of (4.3c) for the upwind scheme is

$$
\phi_{j+1 / 2}^{l}=-\frac{1}{2} \psi\left(a_{j+1 / 2}^{l}\right)\left(g_{j+1}^{l}+g_{j}^{l}\right)+\psi\left(a_{j+1 / 2}^{l}+\gamma_{j+1 / 2}^{l}\right) \alpha_{j+1 / 2}^{l}
$$

with

$$
\begin{gathered}
g_{j}^{l}=\min \bmod \left(\alpha_{j-1 / 2}^{l}, \alpha_{j+1 / 2}^{l}\right), \\
\gamma\left(a_{j+1 / 2}^{l}\right)=\frac{1}{2} \psi\left(a_{j+1 / 2}^{l}\right) \begin{cases}\left(g_{j+1}^{l}-g_{j}^{l}\right) / \alpha_{j+1 / 2}^{l}, & \alpha_{j+1 / 2}^{l} \neq 0 \\
0, & \alpha_{j+1 / 2}^{l}=0 .\end{cases}
\end{gathered}
$$

Numerical studies also show that there is no difference in resolution in using limiter $(3.4 \mathrm{a})$ or $(3.4 \mathrm{~b})$ for the symmetric TVD scheme. Limiter (3.4c) produces slightly sharper shocks than (3.4a) and (3.4b). This conclusion was based on the numerical study for flow field conditions ranging from subcritical to transonic and supersonic for the NACA0012 airfoil. Also, since these test cases consist of shock waves only, the same limiter was used for all characteristic fields. Figures 1 and 2 show a comparison of the current method using limiter (3.4a) with the upwind scheme (5.1) for two inviscid steady-state airfoil calculations. The two solutions are almost indistinguishable. The advantages of symmetric TVD schemes are that they require less computational effort and provide a more natural way of extending the scheme to two and three-dimensional problems. For the current calculations, the upwind TVD scheme requires approximately $35 \%$ more CPU time than the symmetric TVD scheme on the CRAY-XMP computer.
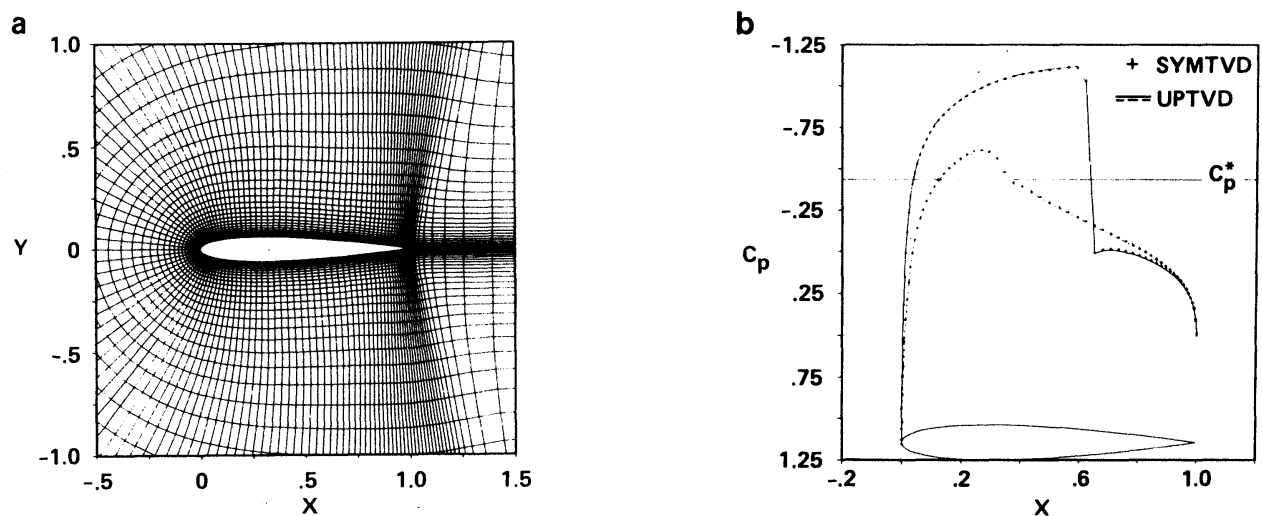

FIG. 1. Comparison of a symmetric TVD (SYMTVD) scheme with an upwind TVD (UPTVD) scheme for the NACA0012 airfoil with $M_{\infty}=0.8, \alpha=1.25^{\circ}$ using a $163 \times 49 C$ grid. 
a

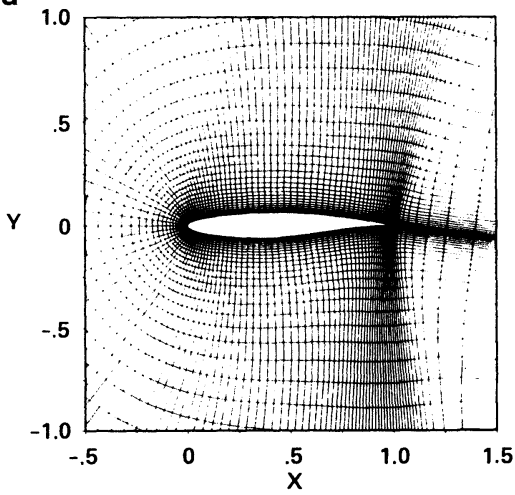

b

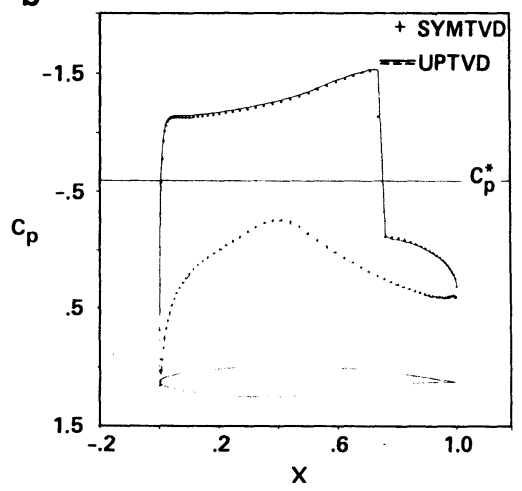

FIG. 2. Comparison of a symmetric TVD (SYMTVD) scheme with an upwind TVD (UPTVD) scheme for the RAE2822 airfoil with $M_{\infty}=0.75, \alpha=3^{\circ}$ using a $163 \times 64 C$ grid.

Figures 3 and 4 show an inviscid comparison of the symmetric TVD scheme with the widely distributed computer code ARC2D, version 150 [20]. The freestream Mach numbers are $M_{\infty}=1.2$ and 1.8 , and the angle of attack is $\alpha=7^{\circ}$. The pressure coefficient distributions (not shown) are identical between the two methods and yet the flow field appears very different. The symmetric TVD scheme gives a very well-ordered flow structure and can still capture the shocks with a coarse grid, especially near the trailing edge of the airfoil. On the other hand, the ARC2D code did rather poorly. The ARC2D, version 150 computer code is based on the Beam and Warming ADI algorithm [22] but uses a mixture of second and fourth-order numerical dissipation terms. These numerical dissipation terms contain adjustable parameters. The values of the parameters in Figs. 3 and 4 are the same value as suggested in reference [20]. Other values of the parameters besides the one used in reference [20] were also studied. What is shown here is representative of the performance of ARC2D for this range of Mach numbers and angles of attack. For subsonic and transonic flow regimes the main advantage of TVD schemes over ARC2D is that one can capture the shock in 1-2 grid points as oppose to 3-4 grids. The flow away from the shock looks very much like ARC2D.

The same problem was studied for the upwind TVD scheme and the results and convergence rates were found to be almost identical to those for the symmetric TVD scheme. For Figs. 3 and 4, a residual of $10^{-12}$ can be reached at around $400-600$ steps. ARC2D, however, required only 200-300 steps to converge to the same residual.

Time-Accurate Calculations: For the time-accurate calculations, the explicit scheme was applied to solve a planar moving shock wave at an angle of attack $30^{\circ}$ and an incident shock Mach number $M_{s}=2$ striking a stationary NACA0018 airfoil. Figure 5 shows the $299 \times 79$ " $C$ " grid used for this problem. The domain of computation is $-2 \leqslant x \leqslant 3,-2.2 \leqslant y \leqslant 2.2$. A detailed description of the problem, 

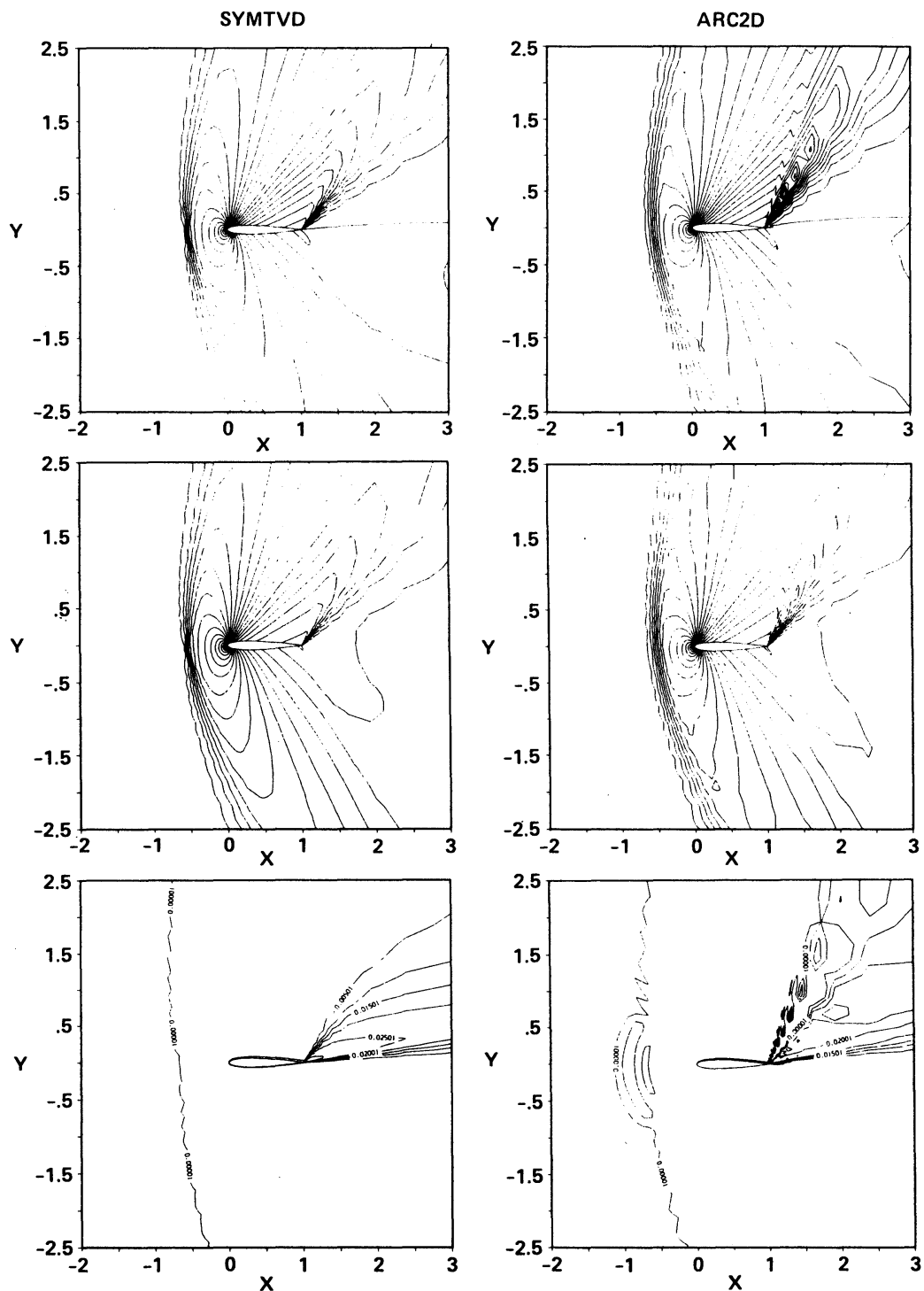

FIG. 3. Comparison of a symmetric TVD (SYMTVD) scheme with ARC2D (version 150) for the Mach contours, pressure contours and entropy contours of the NACA0012 airfoil with $M_{\infty}=1.2, \alpha=7^{\circ}$ using a $163 \times 49 C$ grid as shown in Fig. 1 . 

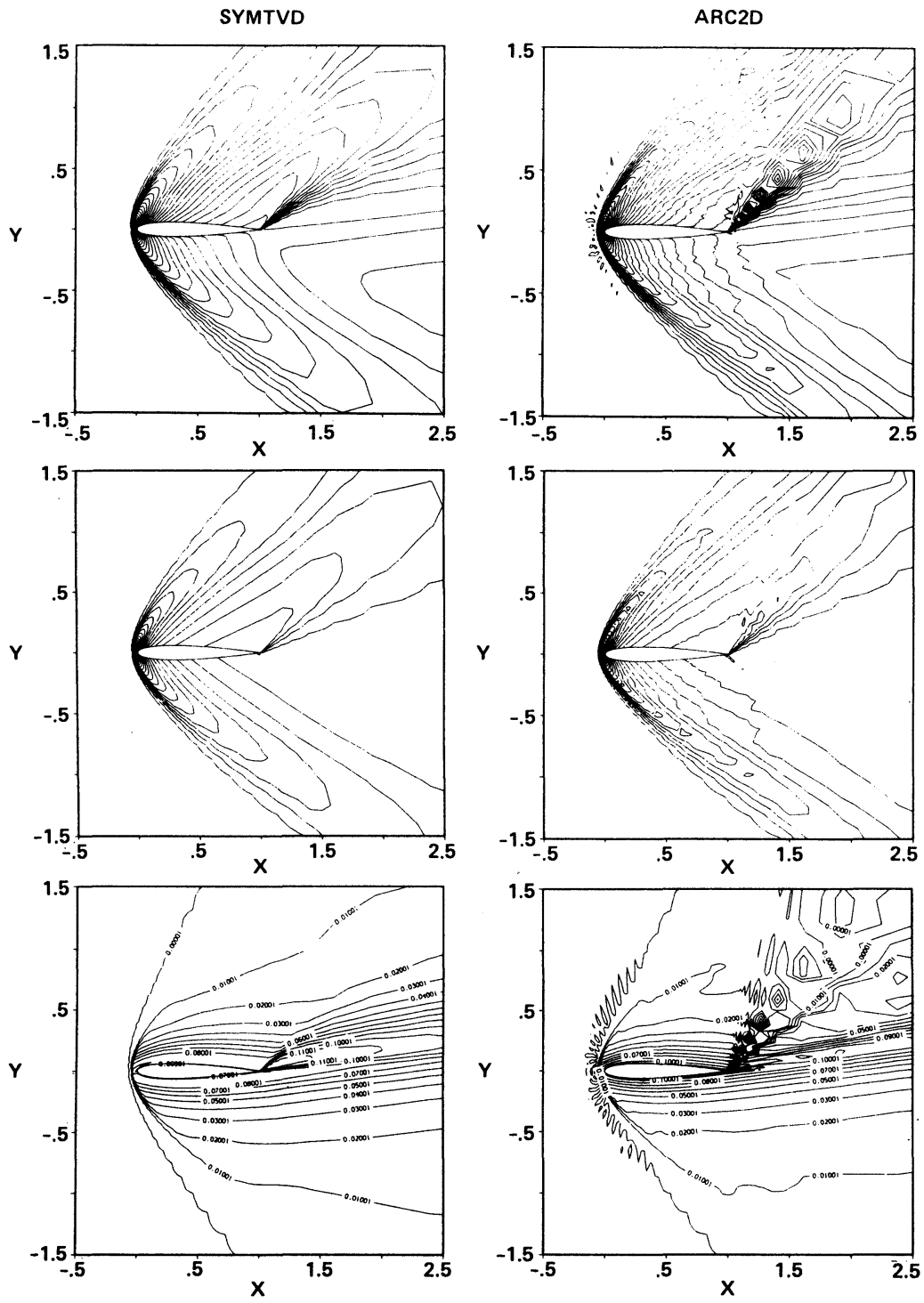

FIG. 4. Comparison of a symmetric TVD (SYMTVD) scheme with ARC2D (version 150) for the Mach contours, pressure contours and entropy contours of the NACA0012 airfoil with $M_{\infty}=1.8, \alpha=7^{\circ}$ using a $163 \times 49 C$ grid as shown in Fig. 1 . 


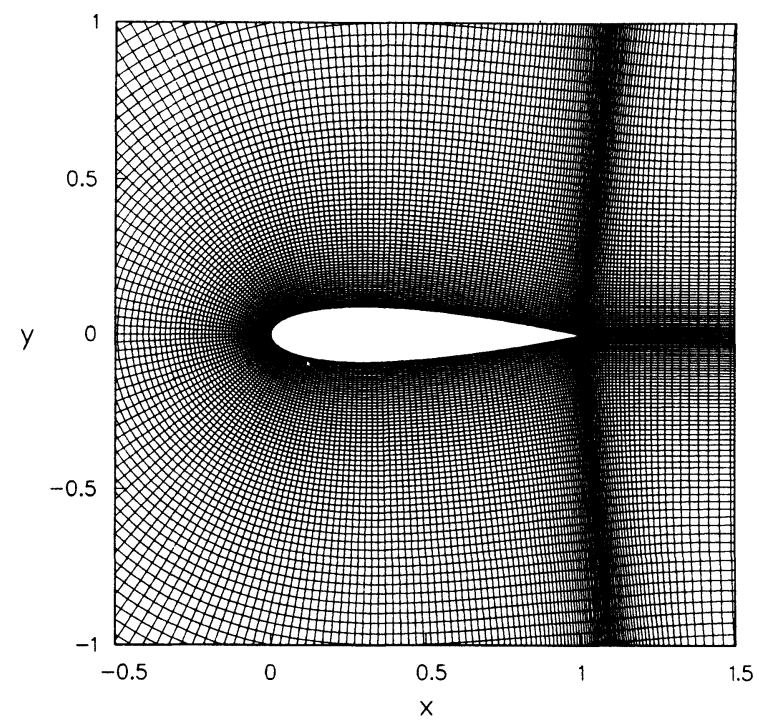

FIg. 5. The $299 \times 79$ " $C$ " grid for the NACA0018 airfoil.

fine grid solution, comparison of numerical solutions with experiments, and comparative study of flux limiters for various types of shock-diffraction problems are reported in references $[23,17]$. Here a comparison of the explicit symmetric TVD scheme using (4.6) with an improved form of the explicit upwind numerical flux (5.1) is illustrated. The $\phi_{j+1 / 2}^{l}$ for the improved second-order in time upwind scheme $[14,17]$ is

$$
\phi_{j+1 / 2}^{l}=-\frac{1}{2} \sigma\left(a_{j+1 / 2}^{l}\right)\left(g_{j+1}^{l}+g_{j}^{l}\right)+\psi\left(a_{j+1 / 2}^{l}+\gamma_{j+1 / 2}^{l}\right) \alpha_{j+1 / 2}^{l},
$$

where

$$
\sigma(z)=\psi(z)-\lambda z^{2},
$$

with

$$
g_{j}^{l}=\frac{\alpha_{j+1 / 2}^{l} \alpha_{j-1 / 2}^{l}+\left|\alpha_{j+1 / 2}^{l} \alpha_{j-1 / 2}^{l}\right|}{\alpha_{j+1 / 2}^{l}+\alpha_{j-1 / 2}^{l}}
$$

for the nonlinear fields, and

$$
\begin{aligned}
& g_{j}^{l}=S \cdot \max \left\{0, \min \left(2\left|\alpha_{j+1 / 2}^{l}\right|, S \cdot \alpha_{j-1 / 2}^{l}\right), \min \left(\left|\alpha_{j+1 / 2}^{l}\right|, 2 S \cdot \alpha_{j-1 / 2}^{l}\right)\right\}, \\
& S=\operatorname{sgn}\left(\alpha_{j+1 / 2}^{l}\right)
\end{aligned}
$$



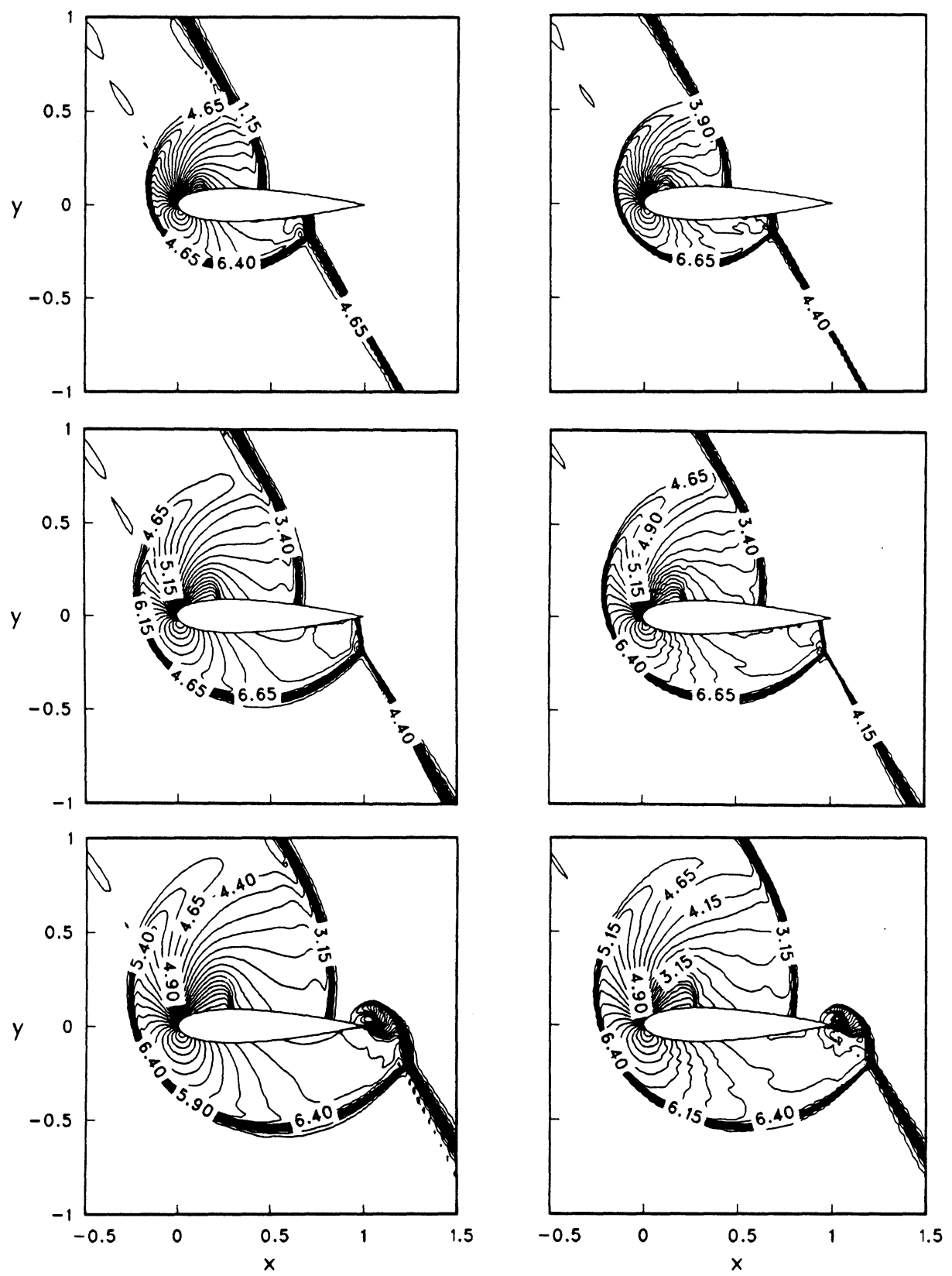

FIG. 6. The pressure contours computed by a symmetric TVD scheme (left) and an upwind TVD scheme (right) for the NACA0018 airfoil with $M_{S}=2, \alpha=30^{\circ}$. 

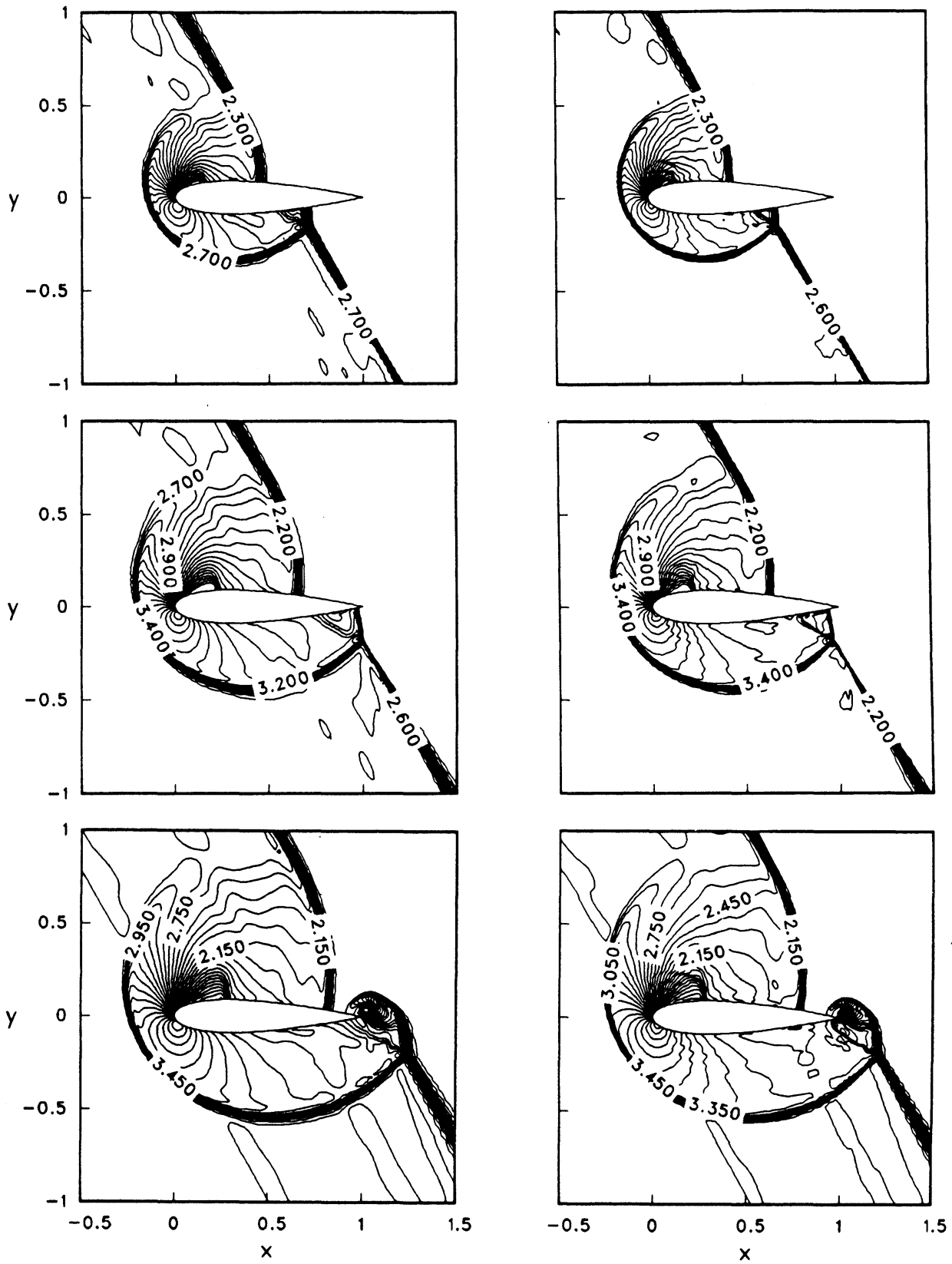

Fig. 7. The density contours computed by a symmetric TVD scheme (left) and an upwind TVD scheme (right) for the NACA0018 airfoil with $M_{S}=2, \alpha=30^{\circ}$. 
for the linear fields, and

$$
\gamma\left(a_{j+1 / 2}^{l}\right)=\frac{1}{2} \sigma\left(a_{j+1 / 2}^{l}\right) \begin{cases}\left(g_{j+1}^{l}-g_{j}^{l}\right) / \alpha_{j+1 / 2}^{l}, & \alpha_{j+1 / 2}^{l} \neq 0 \\ 0, & \alpha_{j+1 / 2}^{l}=0 .\end{cases}
$$

Scheme (5.2) is the same as (5.1) except with different $g_{j}$ and a second-order in time term added. The results in the form of pressure and density contour plots are shown in Figs. 6 and 7 at three different time instances and at approximately the same total time. Both methods used the same limiters and ran at a CFL number of 0.99 . That is, (5.2c) and (5.2d) was used for the upwind method, and (3.4e) for the nonlinear fields and (3.4d) for the linear fields for the symmetric TVD scheme. It can be seen that the symmetric TVD scheme results in a slightly more diffusive pressure flow field and cannot capture the contact surface as well as the upwind TVD scheme. However, the over all agreement is quite good. The present illustration shows only the global shock-diffraction pattern. In order to capture the finer structure of the shock-diffraction pattern, a moving grid with local grid refinement is needed. Aside from CPU time difference, one advantage of the symmetric TVD schemes over the upwind TVD scheme is that symmetric TVD scheme are less sensitive to the numerical boundary condition treatment; see references $[17,18]$ for more detail.

\section{Concluding Remarks}

The present paper was inspired by the work of Roe [11] and Davis [12], and is based on the work of Harten [1,2] and of Harten and the author [7-10]. A oneparameter family of explicit and implicit TVD schemes is reformulated so that a wider group of limiters is included. The current class of schemes as well as Roe and Davis's can be classified as symmetric (or non-upwind) TVD schemes. The main advantages of the present class of schemes over the ones suggested by Osher and Chakravarthy [24], Roe, or Davis are that (a) a wider class of time-differencing is included, (b) the implicit scheme allows a natural linearized procedure for a noniterative implicit procedure, and thus might have a greater potential for practical applications, especially for "stiff" problems, and (c) when applied to steady-state calculations, the numerical solution is independent of the time step. Furthermore, Roe and Davis's formulations can be considered as a member of this family by simply setting $\theta=0$ and using the numerical fluxes (3.7). Extension of this class of schemes and Roe and Davis's schemes to a system of equations is straightforward. One can define a general numerical flux function

$$
H_{j+1 / 2}^{G}=\frac{1}{2}\left(F_{j}+F_{j+1}-R_{j+1 / 2} \Phi_{j+1 / 2}^{G}\right),
$$

where the elements of the $\Phi_{j+1 / 2}^{G}$ denoted by $\left(\phi_{j+1 / 2}^{l}\right)^{G}, l=1, \ldots, m$, are

$$
\left(\phi_{j+1 / 2}^{l}\right)^{G}=\left[\lambda \beta\left(a_{j+1 / 2}^{l}\right)^{2} Q_{j+1 / 2}^{l}+\psi\left(a_{j+1 / 2}^{l}\right)\left(1-Q_{j+1 / 2}^{l}\right)\right] \alpha_{j+1 / 2}^{l},
$$

with $\beta=1$ or 0 for transient calculations, and $\beta=0$ for steady-state calculations. 
Here when $\beta=0,(6.1)$ is $(4.3 \mathrm{~b})$, and when $\beta=1,(6.1)$ is the Roe's Lax-Wendroff numerical flux (4.6).

The results of Roe, Davis, and the present formulation provide a more rational way of supplying additional numerical dissipation terms to the commonly known schemes such as the Lax-Wendroff type and some spatially symmetrical explicit and implicit types of schemes. Here the amount of work required to modify existing computer codes with the suggested numerical dissipation terms varies from very minor changes to moderate yet straightforward computer programming. The potential of improving the robustness and accuracy of a wide variety of physical applications is worth the effort of further pursuing the implementation of these ideas into the many existing computer codes. Numerical experiments with $\beta=0$ and $\theta=1$ for two-dimensional steady-state airfoil calculations, and with $\beta=1, \theta=0$ for two-dimensional blast-wave calculations show the versatility of these schemes.

\section{APPENDIX}

\section{Equivalent Representation for the Conservative Dissipation Term}

The terms $r_{j+1 / 2}^{+}$in (3.2b) are not defined if $\Delta_{j-1 / 2} u$ and $\Delta_{j+3 / 2} u$ are finite and $\Delta_{j+1 / 2} u=0$. To avoid the use of extra logic in a computer implementation, it might be better to rewrite the terms $Q_{j+1 / 2} \Delta_{j+1 / 2} u$ in Eqs. (3.1), (3.8), and thereafter in the form

$$
Q_{j+1 / 2} \Delta_{j+1 / 2} u=\hat{Q}_{j+1 / 2} .
$$

Linear Scalar Hyperbolic Equations. The form $\hat{Q}_{j+1 / 2}$ is a function of $\Delta_{j-1 / 2} u$, $\Delta_{j+1 / 2} u$, and $\Delta_{j+3 / 2} u$, but not $r^{ \pm}$; i.e.,

$$
\hat{Q}_{j+1 / 2}=\hat{Q}\left(\Delta_{j-1 / 2} u, \Delta_{j+1 / 2} u, \Delta_{j+3 / 2} u\right) .
$$

The numerical flux $h_{j+1 / 2}$ in (3.8) can be rewritten as

$$
h_{j+1 / 2}=\frac{1}{2}\left[a\left(u_{j+1}+u_{j}\right)-|a|\left(\Delta_{j+1 / 2} u-\hat{Q}_{j+1 / 2}\right)\right] .
$$

Expression (3.4) now becomes

$$
\begin{aligned}
\hat{Q}\left(\Delta_{j-1 / 2} u, \Delta_{j+1 / 2} u, \Delta_{j+3 / 2} u\right)= & \operatorname{minmod}\left(\Delta_{j+1 / 2} u, \Delta_{j-1 / 2} u\right) \\
& +\operatorname{minmod}\left(\Delta_{j+1 / 2} u, \Delta_{j+3 / 2} u\right)-\Delta_{j+1 / 2} u, \\
\hat{Q}\left(\Delta_{j-1 / 2} u, \Delta_{j+1 / 2} u, \Delta_{j+3 / 2} u\right)= & \operatorname{minmod}\left(\Delta_{j-1 / 2} u, \Delta_{j+1 / 2} u, \Delta_{j+3 / 2} u\right) . \\
\hat{Q}\left(\Delta_{j-1 / 2} u, \Delta_{j+1 / 2} u, \Delta_{j+3 / 2} u\right)= & \operatorname{minmod}\left[2 \Delta_{j-1 / 2} u, 2 \Delta_{j+1 / 2} u, 2 \Delta_{j+3 / 2} u,\right. \\
& \left.\times \frac{1}{2}\left(\Delta_{j-1 / 2} u+\Delta_{j+3 / 2} u\right)\right] \\
\hat{Q}\left(\Delta_{j-1 / 2} u, \Delta_{j+1 / 2} u, \Delta_{j+3 / 2} u\right)= & \operatorname{supb}\left(\Delta_{j+1 / 2} u, \Delta_{j-1 / 2} u\right) \\
& +\operatorname{supb}\left(\Delta_{j+1 / 2} u, \Delta_{j+3 / 2} u\right)-\Delta_{j+1 / 2} u,
\end{aligned}
$$


and

$$
\begin{aligned}
\hat{Q}\left(\Delta_{j-1 / 2} u, \Delta_{j+1 / 2} u, \Delta_{j+3 / 2} u\right)= & \operatorname{vl}\left(\Delta_{j+1 / 2} u, \Delta_{j-1 / 2} u\right) \\
& +\operatorname{vl}\left(\Delta_{j+1 / 2} u, \Delta_{j+3 / 2} u\right)-\Delta_{j+1 / 2} u,
\end{aligned}
$$

In general, the "minmod" function of a list of arguments is equal to the smallest number in absolute value if the list of arguments is of the same sign, or is equal to zero if any argument is of the opposite sign. The function $\operatorname{supb}(\cdot, \cdot)$ and $\operatorname{vl}(\cdot, \cdot)$ are defined as follows:

$$
\begin{aligned}
\operatorname{supb}(x, y) & =\operatorname{sgn}(x) \cdot \max \{0, \min [2|x|, y \cdot \operatorname{sgn}(x)], \min [|x|, 2 y \cdot \operatorname{sgn}(x)]\}, \\
\operatorname{vl}(x, y) & =\frac{x y+|x y|}{x+y} .
\end{aligned}
$$

Nonlinear Scalar Hyperbolic Conservation Laws. For nonlinear problems, one way is to replace all the $a$ 's in equation (A.3) by $a_{j \pm 1 / 2}$ accordingly. The value of $a_{j+1 / 2}$ is defined in (3.14). For $r_{j+1 / 2}^{ \pm}$defined in (3.19), $Q$ should also be redefined as

$$
\hat{Q}_{j+1 / 2}=\hat{Q}\left(\left|a_{j-1 / 2}\right| \Delta_{j-1 / 2} u,\left|a_{j+1 / 2}\right| \Delta_{j+1 / 2} u,\left|a_{j+3 / 2}\right| \Delta_{j+3 / 2} u\right),
$$

and $\Delta_{j \pm 1 / 2} u$ should be replaced by $\left|a_{j \pm 1 / 2}\right| \Delta_{j \pm 1 / 2} u$ wherever they appear in equations (A.4)-(A.6). Similarly, the system case can be rewritten in terms of the $\hat{Q}^{\prime}$ s.

\section{REFERENCES}

1. A. Harten, A High Resolution Scheme for the Computation of Weak Solutions of Hyperbolic Conservation Laws," NYU Report, October 1981; J. Comput. Phys. 49, 357 (1983).

2. A. Harten, On a Class of High Resolution Total-Variation-Stable Finite-Difference Schemes, NYU Report, October, 1982; SIAM J. Numer. Anal. 21, 1 (1984).

3. B. van Leer, J. Comput. Phys. 14, 361 (1974).

4. J. P. Boris AND D. L. Book, J. Comput. Phys. 11, 38 (1973).

5. P. K. SWEBY, SIAM J. Numer. Anal. 21, 995 (1984).

6. P. L. RoE, in Proceedings of the AMS-SIAM Summer Seminar on Large-Scale Computation in Fluid Mechanics, 1983, edited by B. E. Engquist et al. Lectures in Applied Mathematics, Vol. 22 (Amer. Math. Soc., Providence, R. I., 1985), p. 163.

7. H. C. YeE, R. F. Warming, and A. HaRTEN, Implicit Total Variation Diminishing (TVD) Schemes for Steady-State Calculations, AIAA Paper No. 83-1902, Proc. of the AIAA 6th Computational Fluid Dynamics Conference, Danvers, Mass., July, 1983; J. Comput. Phys. 57, 327 (1985).

8. H. C. Yee, R. F. Warming, And A. Harten, in Proceedings of the AMS-SIAM Summer Seminar on Large-Scale Computation in Fluid Mechanics, 1983, edited by B. E. Engquist et al. Lectures in Applied Mathematics, Vol. 22 (Amer. Math. Soc., Providence, R.I., 1985), p. 357.

9. H. C. Yee, Advances in Hyperbolic Partial Differential Equations, a special issue of Int. J. Comput. Math. Appl. 12 A, 413-432 (1986).

10. H. C. YeE ANd A. HaRTen, Implicit TVD Schemes for Hyperbolic Conservation Laws in Curvilinear Coordinates, AIAA Paper No. 85-1513-CP, Proc. of the AIAA 7th Computational Fluid Dynamics Conference, Cinn., Ohio, July 15-17, 1985; AIAA J., in press. 
11. P. L. RoE, Generalized Formulation of TVD Lax-Wendroff Schemes, ICASE Report No. 84-53, October 1984 (unpublished).

12. S. F. DAVIs, TVD Finite Difference Schemes and Artificial Viscosity, ICASE Report No. 84-20, June 1984 (unpublished).

13. H. C. YeE, in Proceedings of the 6th GAMM conference on Numerical Methods in Fluid Mechanics, Gottingen, West Germany, September 25-27, 1985; Notes on Numerical Fluid Mechanics, Vol. 13, Veiweg, West Germany.

14. H. C. YeE, Improved Explicit and Implicit TVD schemes for Multidimensional Compressible Gas Dynamics Equation, in preparation.

15. H. C. YeE, On the Implementation of a Class of Upwind Schemes for System of Hyperbolic Conservation Laws, NASA-TM-86839, September 1985.

16. A. TADMOR, Math. Comput. 43, 369 (1984).

17. H. C. YeE, A Comparative Study of Flux Limiters for Two-Dimensional Time-Accurate Calculations, in preparation.

18. H. C. YeE, in Proceedings of the 10th International Conference on Numerical Methods in Fluid Dynamics, June 23-27, 1986, Beijing, China.

19. P. K. Sweby, High Resolution Schemes Using Flux Limiters for Hyperbolic Conservation Laws, U.C.L.A. Report, June 1983, Los Angeles, Calif. (unpublished).

20. T. H. Pulliam and J. Steger, Recent Improvements in Efficiency, Accuracy and Convergence for Implicit Approximate Factorization Algorithms, AIAA Paper No. 85-0360, 1985 (unpublished).

21. A. Jameson, W. Schmidt, AND E. Turkel, Numerical Solutions of the Euler Equations by Finite Volume Methods Using Runge-Kutta Time-Stepping Schemes, AIAA Paper No.81-1259, 1981 (unpublished).

22. R. M. Beam and R. F. Warming, J. Comput. Phys. 22, 87 (1976).

23. Y. J. Moon AND H. C. YeE, Numerical Simulation by TVD Schemes of Complex Shock Reflections from Airfoils at High Angle of Attack, AIAA 25 th Aerospace Science Meeting, January 12-15, 1987, Reno, Nevada.

24. S. Osher AND S. Chakravarthy, SiaM J. Numer. Anal. 21, 955 (1984). 